\title{
Multidiagnostics investigation of the role of the magnetic field profile in a simple mirror trap
}

\author{
G. Castro, D. Mascali, and M. Mazzaglia \\ INFN Laboratori Nazionali del Sud, via S. Sofia 62, 95123 Catania, Italy \\ S. Briefi and U. Fantz \\ Max-Planck-Institut für Plasmaphysik, Boltzmannstrasse 2, 85748 Garching, Germany \\ R. Miracoli \\ ESS Bilbao, Laida Bidea Edificio 207-B, 48160 Derio, Spain
}

(Received 29 March 2019; published 31 May 2019)

\begin{abstract}
The impact of the magnetic field profile on the operations of plasma-based Electron Cyclotron Resonance Ion Sources (ECRIS) has been debated and investigated for a long time, and a summary is given by the collection of the so-called "magnetic scaling laws", i.e., some rules-of-thumb providing the optimal configuration of the axial and radial confining magnetic fields. Anyway, the optimal configurations have been found just looking at the output beam currents and charge states. Only recently, more advanced experiments have shown that the optimal performances are related to some "islands" in the parameters space leading to a dense, energetic but stable plasma. A general, systematic characterization of plasma density and temperature as a function of the magnetic scaling, and for different energy domains (i.e., for cold, warm, and hot electrons) is not yet available. This paper presents a multidiagnostics characterization of a hydrogen plasma heated by microwaves at around $6.8 \mathrm{GHz}$ via Electron Cyclotron Resonance, and confined in an axisymmetric simple mirror magnetic trap. Investigation of plasma response to solely the axial field profile gives the unique opportunity to decouple the effect of simple mirror field from the radial components that are, usually, provided by a hexapole. Optical emission spectroscopy, Langmuir probe, and an x-ray silicon drift detector were simultaneously used to evaluate the plasma density and temperature in different energy domains, thus exploring the electron energy distribution function from a few $\mathrm{eV}$ to hundreds of $\mathrm{keV}$. Plasma parameters have been measured as a function of the magnetic field profile (in particular by varying the ratio $B_{\min } / B_{\mathrm{ECR}}$ ), of the microwave power and of the neutral pressure. Furthermore, for the same scaling, the relative abundances of $H$ atoms and $H_{2}$ have been estimated. Data show smooth trends of electron density and temperatures versus microwave power and neutral pressure, but strong and even nonlinear responses as a function of the magnetic field profile variation, allowing us to connect semiempirical scaling laws to changes in plasma parameters.
\end{abstract}

DOI: 10.1103/PhysRevAccelBeams.22.053404

\section{INTRODUCTION}

Over the last years, the requirements posed on ion sources used for feeding particle accelerators have increased steadily, for both positive and negative ion sources. Ions are typically created in a low pressure plasma source. In order to perform a detailed performance optimization of ion sources, knowledge about plasma parameters is inevitable. Therefore, dedicated efforts concerning the characterization of source plasma have been carried out.

Published by the American Physical Society under the terms of the Creative Commons Attribution 4.0 International license. Further distribution of this work must maintain attribution to the author(s) and the published article's title, journal citation, and DOI.
One example is the negative hydrogen ion source applied at the Linac4 accelerator at CERN. For this source, a combined approach between optical emission spectroscopy measurements and numerical modeling has been successfully taken and detailed insights in the plasma processes obtained [1]. In contrast to the plasma generation via inductive microwave coupling applied at the Linac4 source, many other sources, especially those dedicated to the production of intense proton beams or multiply-charged ions, are based on ECR coupling utilizing microwaves in the $\mathrm{GHz}$ range. These devices are the so-called ECRIS: Electron Cyclotron Resonance Ion Sources. Since multiple ionization is a stepwise process, lasting for hundreds of $\mathrm{ms}$ in case of heavy ions, these machines need plasma confinement provided by simple or multimirror magnetic traps (where the magnetic field strengths lie in the 
range $0.1-4 \mathrm{~T})$. Conditions for the stripping of heavy ions are really demanding in terms of plasma density, temperature and confinement times: estimated plasma densities lie in the range $n_{e} \sim 10^{17}-10^{20} \mathrm{~m}^{-3}$, and temperatures $T_{e} \sim$ $0.1-30 \mathrm{keV}$, while ion lifetime must be larger than $0.1 \mathrm{~s}$. For sake of compactness (the cylindrical plasma chambers are fully surrounded by magnetic systems yokes, while the endplates host gas and microwaves injection systems and the ions extraction electrode) historically only a very limited number of diagnostics have been applied to ECRIS plasmas. Therefore, in most of the cases, plasma properties such as density and temperature were only known via indirect methods (e.g., from the analysis of extracted ion beam currents and $m / q$ spectra [2]), or estimated from semiempirical considerations. Since the early 2000s, however, several research teams started to develop standard methods of plasma diagnostics to be applied in the ECRIS field. X-ray detectors were formerly used in USA (in particular in Berkeley, at LNBL) to characterize fluxes of hard-x-rays emitted from the VENUS source [3,4], measuring radiation produced by very hot electrons in the plasma (up to $2 \mathrm{MeV}$ ). Langmuir probes, used by some groups to estimate bulk plasma density, temperature, and potential $[5,6]$, were limited to low-frequency devices, producing plasmas at moderate energy contents. In 2002, at the Hungarian Academy of Science, a first experiment employing $\mathrm{x}$-ray pin-hole cameras-enabling space resolved $\mathrm{x}$-ray spectroscopywas carried out [7]. About this latter methodology, research continued producing new results concerning the measurements of soft- $X$ radiation emitted by the so-called "warm electrons" $(2<E<30 \mathrm{keV})$, and the x-ray fluorescence produced by the inner plasma ions such as $\operatorname{Ar}\left(K_{\alpha}\right.$ line at $2.96 \mathrm{keV}$ ) [8,9]. More recently, at Jyvaskyla University, Finland, researchers developed optical emission spectroscopy (OES) for measuring ECRIS plasma ion temperature [10].

\section{A. Understanding physical meaning of magnetic scaling laws in ECRIS}

Currently, the parameter space is still not fully known, especially in terms of plasma densities and temperatures in the multicomponent plasma under different discharge configurations. Especially, still debated is the global scaling of plasma parameters as a function of tuning parameters, namely pumping wave power and frequency, background neutral pressure and magnetic field profiles. The latter plays a particularly critical role, and its impact has been explained in most of cases through semiempirical scaling laws, whose physical basis are still debated. The shortest summary of them says that in a multimirror trap such as B-minimum configurations (given by the superposition of a simple mirror configuration plus a radial field component provided by an hexapole) the axial and radial mirror ratios must obey to the law $B_{\text {rad }} / B_{\min } \simeq B_{\max } / B_{\min } \geq 2$.
This relation comes from considerations about magnetohydrodynamics stability, theoretically and experimentally proven [11] and nowadays widely used worldwide in routine operations of ECR Ion Sources.

However, several empirical observations about output beam current dependence on the magnetic field tuning leaded to additional scaling rules, currently poorly motivated by theory, models or direct observation of plasma parameters. This exhaustive set of magnetic laws were derived in [12]: $B_{\text {rad }} \geq 2 B_{\mathrm{ECR}}, B_{\mathrm{inj}} \simeq 3 B_{\mathrm{ECR}}$ or more, $B_{\mathrm{ext}} \simeq B_{\mathrm{rad}}$, $0.30<B_{\min } / B_{\text {rad }}<0.45, \quad 0.65<B_{\min } / B_{\mathrm{ECR}}<0.75$; where $B_{\text {inj }}, B_{\text {ext }}, B_{\min }, B_{\text {rad }}, B_{\mathrm{ECR}}$ are the B values at the injection and extraction endplate, the minimum longitudinal value and the maximum radial field, and the field corresponding to the cyclotron resonance, respectively. Particularly interesting is the case of $B_{\min } / B_{\mathrm{ECR}}$ scaling. In ECRIS devoted to the production of multiply-charged ions, for instance, it has been observed that even a very fine increase of such ratio (not enough to significantly affect the confinement strength, however), and especially when it overcomes values around $0.75-0.78$, that can be considered as a critical value, causes a sudden and nonlinear increase of the plasma $\mathrm{x}$-ray emission; this is then correlated with a drop of ion beam currents, especially for the highest charge states [11]. Consequently, optimal operative conditions for routine ECRIS operation are fixed below this threshold. Very few information exists concerning the role played by the "bulk" plasma, made of cold electrons (few $\mathrm{eV}$ of temperature) and by the overall electron energy distribution function (EEDF), during this scaling. It is worth mentioning that the complete set of scaling has been obtained along the years by looking to the output beam properties only, namely ion current and average charge state. A complete characterization of plasma response to the several scaling of the magnetic trap has never been performed, due to the substantial difficulties mentioned above. Recently, experiments have shown that the overcome of the critical value in the $B_{\min } / B_{\mathrm{ECR}}$ ratio may be a condition necessary (even if not sufficient since rf power and background pressure play a role too) to the onset of the CyclotronMaser instabilities $[13,14]$. This evidence has been supported by measurements of the plasma self-produced radio waves released in a burst lasting few hundreds of ns, followed by $\mathrm{x}$-ray emission in a timescale of several $\mathrm{ms}$. Now we deem that a quantitative time-integrated analysis of the plasma properties could shed additional light on the axial field scaling, in particular about its impact on plasma density and temperature.

\section{B. Goal of the paper}

In this paper, a comprehensive diagnostic characterization of an ECR plasma confined in a simple mirror magnetic trap is presented. The main goal is to investigate the response of electron density and temperature as a function of the applied external magnetic field, of microwave power and, eventually, of gas pressure. In particular, the variation of electron density and temperature will be 
investigated when varying the axial profile of the magnetic field, and especially the ratio $B_{\min } / B_{\mathrm{ECR}}$. Following the discussion in the previous section, these results are particularly relevant to explore the effects of solely the axial field, since in ECR ion sources the confinement is also supported by the radial sextupolar component of the magnetic field.

Since the measurements have been carried out in higher pressure condition $\left(\sim 10^{-4}\right.$ mbar versus $\sim 10^{-6}-10^{-7}$ mbar in ECRIS during daily operation), and no radial confinement is present in our set-up, absolute value of density and temperature cannot be compared to those of operating ECRIS. However, several experiments show that the magnetic scaling remains valid even at higher pressure ranges (above $10^{-3}$ mbar).

The experimental results about density and temperature of the bulk plasma come from the simultaneous use of a spatially movable Langmuir Probe (LP) compared with the line-integrated information collected by an optical emission spectrometer; the effect of the $B_{\min } / B_{\mathrm{ECR}}$ ratio at its higher values will be benchmarked by $\mathrm{x}$-ray spectroscopy, in terms of $\mathrm{x}$-ray emission rate and estimated spectral temperature in the soft-X domain.

\section{EXPERIMENTAL SETUP AND DIAGNOSTIC METHODS}

\section{A. The flexible plasma trap}

The flexible plasma trap (FPT) test-bench has been designed, developed and commissioned at INFN-LNS with the specific aim to enclose in a simple and flexible ECRISrelevant device all instruments required for a detailed plasma characterization. FPT consists of a cylindrical discharge vessel made of stainless steel with a radius of $4.1 \mathrm{~cm}$ and a length of $26.0 \mathrm{~cm}$. A sketch of the FPT setup is shown in Fig. 1. The external magnetic field used for ECR heating and plasma confinement is generated by three solenoids which also allow tuning the magnetic profile. In particular, a simple magnetic mirror, a constant B field along the axis of the cylindrical discharge vessel and a

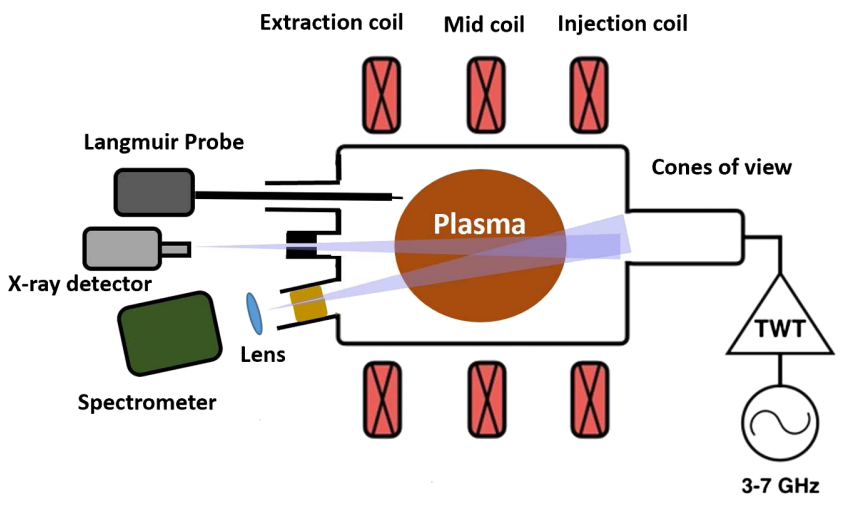

FIG. 1. Sketch of the FPT setup including the arrangement of the diagnostics.

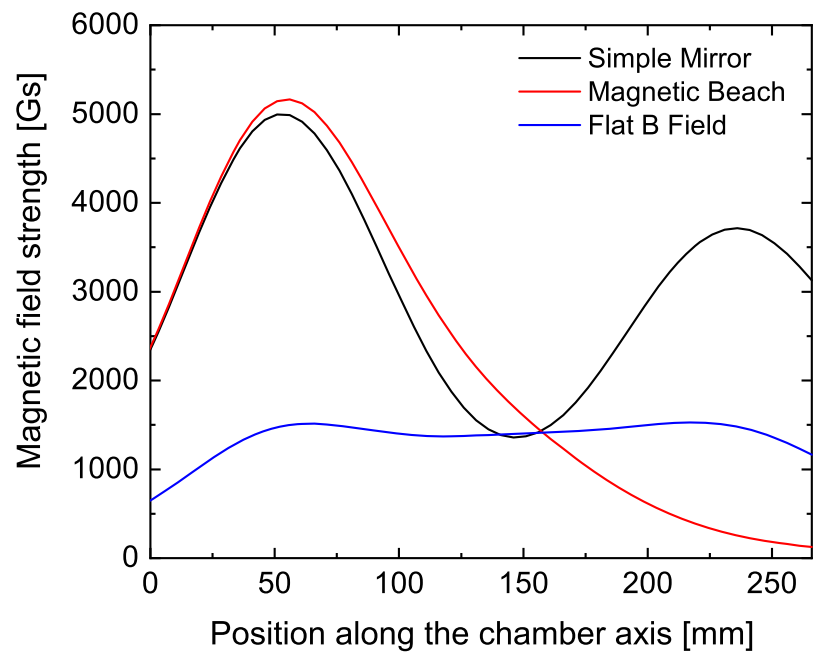

FIG. 2. Magnetic field configurations that can be realized at FPT: constant B field along the chamber axis, simple mirror and magnetic beach. In this plot, the magnetic field is evaluated on the plasma chamber axis.

magnetic beach configuration can be generated by tuning the current flowing in the coils (see Fig. 2). A characterization of FPT performances in B-flat configuration can be found in [15]. In this work, we focused on the investigation of the simple mirror, varying the $B_{\min } / B_{\max }$ ratio.

Microwaves are generated by a traveling wave tube (TWT) operating in a range from 4 to $7 \mathrm{GHz}$. Operative values of microwave power are in the range 0-300 $\mathrm{W}$ while the hydrogen pressure is typically between $5 \times 10^{-5}-5 \times 10^{-3}$ mbar. Further information about the FPT can be found in Ref. [16].

\section{B. Langmuir probe diagnostics}

Plasma immersed electrostatic probes represent the easiest way to perform the measurements of density and temperature of low energy plasma electrons. Information about the EEDF in the low energy range and about the plasma potential can also be obtained. At FPT, the Langmuir probe (LP) performs measurements along a penetration axis parallel to the plasma chamber axis, as shown in Fig. 1. It can also be moved in the axial direction in order to record profiles of the plasma parameters. The probe tip consists of a tungsten wire having a length of $4 \mathrm{~mm}$ and a diameter of $125 \mu \mathrm{m}$.

Evaluation of plasma parameters by resistivity curve depends on the theoretical model applied. Several models have been developed through the years. Any LP model shows a characteristic validity range, depending on the ratio between probe radius and Debye length, ion temperature, and collision frequency. The presence of a strong magnetic field leads to a strong anisotropy in the electron motion and makes the electron component of the resistivity curve useless for diagnostics. On the other side, it is well known 
the ion component is almost unaffected by the magnetic field and can be used for the plasma parameters calculation. Consequently, we used the ion-part only from the I-V curve in all LP measurements, and also we hypothesized that the electron density $n_{e}$ was equal to the ion density $n_{i}$, i.e., the mean charge state to be one. In the density range expected for the FPT plasma $\left(10^{16}-10^{17} \mathrm{~m}^{-3}\right)$, the classical and well-known orbital motion limited (OML) model [17] could overestimate the density values [18]. Hence, in this paper, electron density has been calculated as the average between the value estimated by the Allen, Boyd, and Reynolds (ABR) model [19] and the Bernstein, Rabinowitz, and Lafambroise (BRL) model [20] as suggested by Chen [21]. Finally, the electron temperature has been calculated by using the BRL model.

It should be noted that the Langmuir probe is an invasive diagnostic, i.e., the presence of the probe can locally perturb, even relevantly, the plasma. Especially for ECR sources, where the characteristic scale-length of plasma chamber, magnetic field, and density gradients lie in the range of $\mathrm{cm}$, the insertion of the probe has been proven to have a critical influence on the microwave-plasma coupling. This can lead deviating plasma parameters during the measurement compared to the undisturbed case. Indeed, investigations performed at the FPT have shown that the probe influences the coupling: when the Langmuir probe is moved into the plasma chamber, the value of the reflected microwave power increases, reducing the net power in the chamber and therefore the electron density in the plasma. The magnitude of this distortion is less pronounced for higher plasma densities as, in such a case, plasma response to microwave propagation tends to dominate with respect to the LP induced perturbations [22]. To account for this perturbation, the results collected via LP have been always normalized to the net forward power.

\section{Optical emission spectroscopy}

A method for determining discharge parameters in a noninvasive way is provided by optical emission spectroscopy (OES). However, this diagnostic has the drawback that only line-of-sight-integrated results are obtained. Spectroscopic measurements have been carried out at the FPT source with an intensity calibrated survey spectrometer ( $\Delta \lambda_{\mathrm{FWHM}} \approx 1 \mathrm{~nm}$ ) for the Balmer series of atomic hydrogen $\left(\mathrm{H}_{\alpha}\right.$ to $\left.\mathrm{H}_{\gamma}\right)$ as well as for the Fulcher- $\alpha$ transition of the $\mathrm{H}_{2}$ molecule $\left(d^{3} \Pi_{u} \rightarrow a^{3} \Sigma_{g}^{+}\right)$. Figure 1 shows the line of sight (diameter limited to $4 \mathrm{~mm}$ by an aperture stop positioned in front of the light collecting optics) where the OES measurements have been performed. A detailed evaluation of the Fulcher- $\alpha$ emission spectrum including an assessment of the ro-vibrational population [23] was not possible due to the low spectral resolution. In order to obtain a representative number for the intensity of this molecular transition, the plasma emission has been integrated in the most intense part of the Fulcher- $\alpha$ transition between 600 and $640 \mathrm{~nm}$.

The measured emissivities have been evaluated with the collisional radiative (CR) models Yacora $\mathrm{H}$ and Yacora $\mathrm{H}_{2}$ [24]. These models balance all relevant population and depopulation processes for the particular states in the hydrogen atom or molecule respectively, thereby yielding steady state population densities. Processes including the reabsorption of photons are not relevant for describing the plasma of the FPT due to the low pressure which means the plasma emission is optically thin. As input parameters for the models the densities and temperatures of the neutral $\left(\mathrm{H}\right.$ and $\mathrm{H}_{2}$ ) and charged particles (electrons and $\mathrm{H}^{+}, \mathrm{H}_{2}^{+}$, $\mathrm{H}_{3}^{+}, \mathrm{H}^{-}$) are required in general. In order to determine plasma parameters from OES measurements, these input parameters are varied until both the absolute emissivities and the line intensity ratios of the measurements are matched thereby yielding the particle densities. For the investigated discharges, the population is dominantly caused by processes involving atomic or molecular hydrogen whereas the hydrogen ions play only a very minor role. This eliminates the possibility of determining the densities of $\mathrm{H}^{+}, \mathrm{H}_{+}, \mathrm{H}_{3}^{+}$, and $\mathrm{H}^{-}$reliably but it also facilitates the OES evaluation as the number of free parameters is reduced considerably to $T_{e}, n_{e}, n_{H}$, and $n_{H_{2}}$. Concerning $T_{\mathrm{gas}}$, which is required as another input parameter for the CR models, the Fulcher- $\alpha$ spectrum has been recorded with a nonintensity calibrated scanning spectrometer having a higher resolution than the survey spectrometer. From this measurement, an estimate of the gas temperature of about $500 \mathrm{~K}$ could be determined.

It should be kept in mind that only a representative integral could be taken for the emissivity of the Fulcher- $\alpha$ transition. Therefore, the density ratio of atomic to molecular hydrogen which is primarily obtained from the intensity ratios between the atomic and molecular emission must be considered as a rough estimate.

\section{X-ray diagnostics}

The $\mathrm{x}$-ray volumetric measurement is a powerful method for the determining the plasma density and temperature of the warm/hot (i.e., above $500 \mathrm{eV}$ and up to the hard $\mathrm{x}$-ray spectrum, lying in the hundreds of keVs domain) component of the EEDF. However, it requires a proper collimation of the x-ray flux for fixing the solid angle covered by the $\mathrm{x}$-ray detector in addition to the use of an adequate emissivity model for the data evaluation. Typically, ECRIS plasmas are characterized by nonisotropic EEDFs due to the cyclotron acceleration what results in anisotropic $\mathrm{x}$-ray emission. However, for sake of simplicity, we follow the approach described in Refs. [8,25,26] which assumes fully isotropic emission. This procedure would enable us to determine both the electron temperature and the density of electrons emitting in the soft x-ray domain. Unfortunately, since the cone of view of the x-ray detector partially 
intercepts the plasma chamber walls (this problem cannot be avoided in the current setup), it was not possible to clearly separate the emission from the plasma (due to internal Bremsstrahlung of the warm/hot electrons) from the Bremsstrahlung radiation coming from the chamber walls, due to the deconfined electrons. Therefore, the absolute value of the electron density in the plasma cannot be determined reliably, and the electron temperature only will be included in the discussion hereinafter. Nevertheless, comparing the relative $\mathrm{x}$-ray emission rate in the different plasma configurations one can give a rough estimation about the total number of electrons contained in the plasma and emitting in the x-rays domain. During the experimental campaign, a silicon drift $\mathrm{x}$-ray detector (SDD) was placed beyond a $8 \mu \mathrm{m}$ Kapton foil for vacuum break and collimated by a lead cylinder with a drilled hole having a diameter of $1 \mathrm{~mm}$ and a length of $60 \mathrm{~mm}$. This set-up was sensitive to the fraction of $\mathrm{x}$-rays within the energy range 2.5-30 keV; detection of x-ray emission from the plasma was collimated within a cone of view of around $4.5^{\circ}$.

\section{EXPERIMENTAL RESULTS}

During the experimental campaign, the largest part of measurements has been carried out in a simple mirror configuration. Input microwave frequency has been set at $6.83 \mathrm{GHz}$, a value which was proved to optimize the wave-toplasma coupling (low reflected power). The injection and extraction coil currents have been fixed at 440 and 411 A respectively, while the one in the midcoil has been changed from $-88 \mathrm{~A}$ (the minus means that current flows inversely to that of injection and extraction coils) to $+60 \mathrm{~A}$. The change in mid coil current allowed to modify the $B_{\min } / B_{\mathrm{ECR}}$ along the chamber axis from $0.56\left(I_{\text {midcoil }}=-88 \mathrm{~A}\right)$ to 1.04 $\left(I_{\text {midcoil }}=+60 \mathrm{~A}\right)$. Figure 3 shows a selection of the different magnetic profiles characterized together with $B_{\mathrm{ECR}}$.

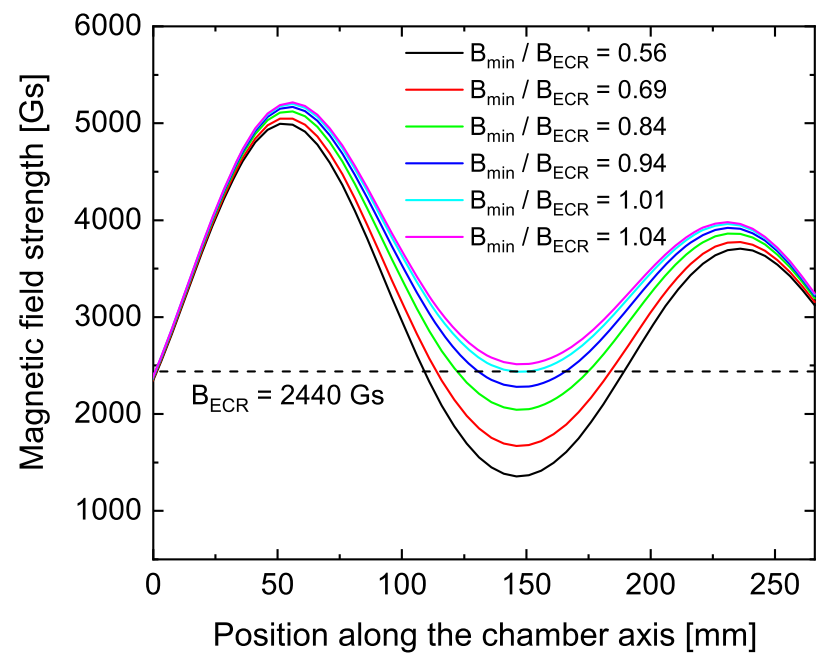

FIG. 3. Magnetic field profile along the plasma chamber axis when increasing the $B_{\min } / B_{\mathrm{ECR}}$ ratio.

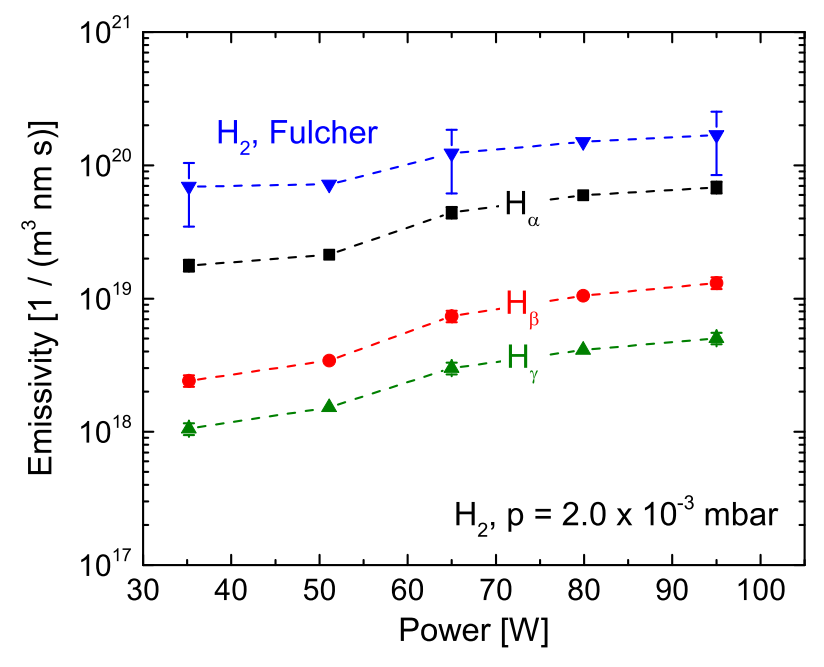

FIG. 4. Emissivities of the atomic Balmer lines $\left(\mathrm{H}_{\alpha}\right.$ to $\left.\mathrm{H}_{\gamma}\right)$ and of the integral between 600 and $640 \mathrm{~nm}$ taken as representative for the molecular Fulcher transition for a variation of the microwave power at fixed pressure of $2.0 \times 10^{-3} \mathrm{mbar}$ and fixed ratio $B_{\min } / B_{\mathrm{ECR}}=0.56$.

\section{A. Influence of pressure and microwave power on plasma parameters}

In a first step, we characterized the $B_{\min } / B_{\mathrm{ECR}}=0.56$ configuration for different value of pressure and microwave power. Figure 4 shows the measured emissivities of the atomic Balmer and the molecular Fulcher radiation exemplary for a pressure of $2 \times 10^{-3}$ mbar. It can be seen that for increasing power all emissivities increase as well; this trend was observed at all the pressures. Evaluation of electron density and temperature via CR models (as explained in Sec. II C) helps to explain these experimental evidences. Figure 5 summarizes the obtained values when varying

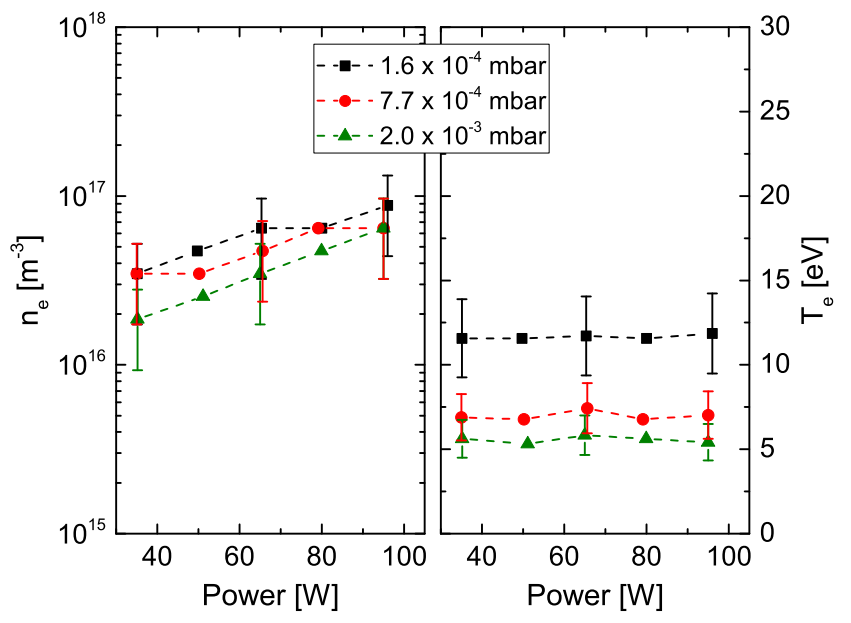

FIG. 5. Electron densities and temperatures obtained from the OES evaluations for varying power at different pressures and fixed ratio $B_{\min } / B_{\mathrm{ECR}}=0.56$. 
microwave power at a pressure of $1.6 \times 10^{-4} \mathrm{mbar}$, $7.7 \times 10^{-4} \mathrm{mbar}$, and $2 \times 10^{-3} \mathrm{mbar}$. As expected, the electron density increased at higher microwave power levels, whereas the electron temperature remains more or less constant. Comparing the different pressures, on the average the $T_{e}$ values are larger at lower pressures, whilst $n_{e}$ does not show any appreciable variation with the pressure, at least within the error bars. This last result is difficult to explain unless one assumes that the ionization fraction at these power levels is mostly dominated by the magnetic confinement and by the microwave power itself. The increase of the emissivity with microwave power can be explained by $n_{e}$ growth.

Figure 6 shows the density ratios of atomic to molecular hydrogen for varying power for the same three pressures as above. They show an increase with higher power which can again be explained by the increase in $n_{e}$ what leads to a higher dissociation rate. As expected, for fixed values of microwave power, $n_{H} / n_{H_{2}}$ decreases when the pressure increases. This trend can be attributed to the decreasing electron temperature leading to a lower dissociation rate (the electron density stays virtually constant at varying pressure).

Figure 7 shows the electron density and temperature profiles within the plasma chamber obtained at a pressure $1.6 \times 10^{-4}$ mbar and $80 \mathrm{~W}$ microwave power measured by LP. It can be seen that the plasma is confined within the ECR layer by the simple mirror trap. A slight increase of electron density at around $250 \mathrm{~mm}$ along the chamber axis is found. The cause of such density increase is not clear at all; it could be due to some plasma formation induced by the LP presence. Electron temperature is around $10 \mathrm{eV}$ within the ECR layer, but shows an isolated maximum of $15 \mathrm{eV}$ in correspondence of the ECR layer.

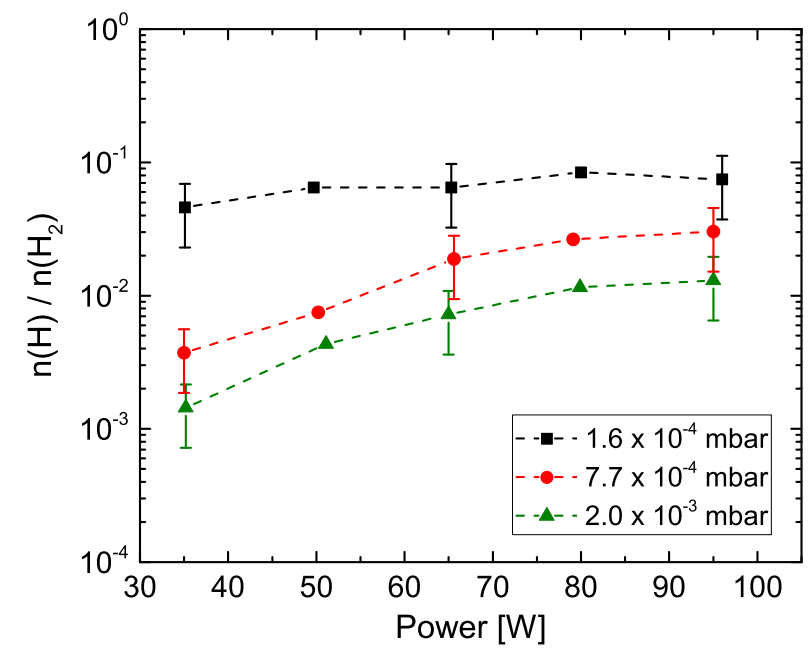

FIG. 6. Density ratio of atomic to molecular hydrogen obtained from the OES evaluations for varying power at different pressures and fixed ratio $B_{\min } / B_{\mathrm{ECR}}=0.56$.

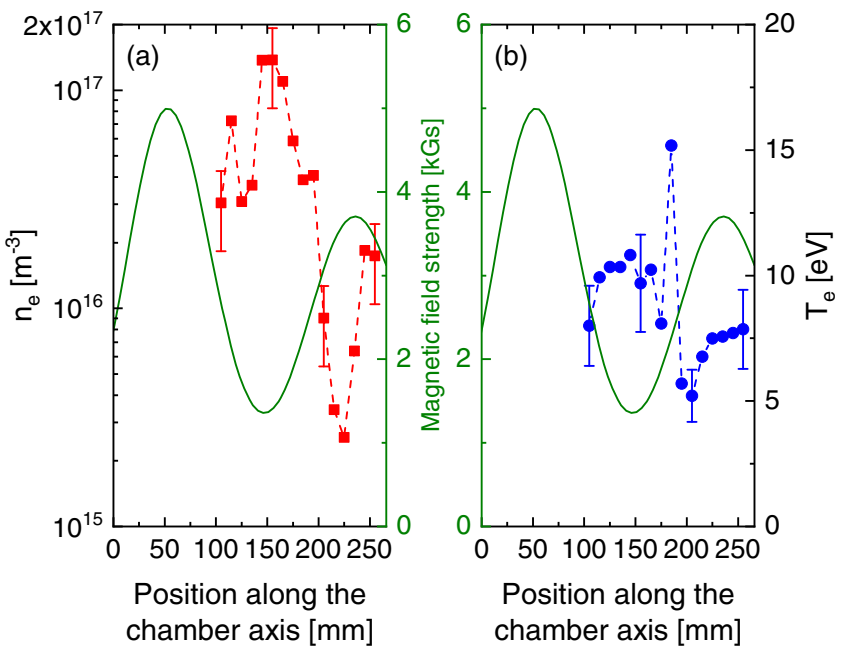

FIG. 7. Electron density (a) and temperature (b) profiles determined from the LP for $B_{\min } / B_{\mathrm{ECR}}=0.56$ at a pressure of $1.6 \times 10^{-4}$ mbar and $80 \mathrm{~W}$ microwave power.

\section{B. Influence of the magnetic field on plasma parameters}

The influence of the magnetic profile on plasma parameters has been characterized by means of OES, LP and $\mathrm{X}$-ray diagnostics. $B_{\min } / B_{\mathrm{ECR}}$ has been changed from 0.56 to 1.04 , with steps of $0.04-0.05$, for two different values of pressure: $1.5 \times 10^{-4}$ and $2.0 \times 10^{-3}$ mbar. Microwave power has been kept constant at $80 \mathrm{~W}$. A comparison between LP and OES diagnostics can be done only by averaging the values of electron density and temperature coming from LP measurements, since OES data are intrinsically the result of a line-of-sight average. Figure 8 shows the trend of average electron density and temperature versus $B_{\min } / B_{\mathrm{ECR}}$ for two pressure values (full symbols). The electron density is, for any value of microwave power and pressure, lower than the cutoff density, which is $5.3 \times 10^{17} \mathrm{~m}^{-3}$ at the operative frequency. The maximum electron density is obtained when $0.65<$ $B_{\min } / B_{\mathrm{ECR}}<0.84$ with an absolute maximum at around 0.7 (pressure: $1.5 \times 10^{-4} \mathrm{mbar}$ ) and 0.75 (pressure: $2.0 \times 10^{-3}$ mbar). These values are in total accordance with a typical rule of thumb of ECR ion sources, fixing the scaling laws for the ratios of the magnetic fields minima and maxima in the trap as explained in Sec. I A. Comparing these results to the electron density obtained from OES, a shift in the maximum $n_{e}$ values towards $B_{\min } / B_{\mathrm{ECR}}<0.7$ can be observed. For low pressure, the absolute values of OES and LP agree reasonably within the error bars whereas at a pressure of $2.0 \times 10^{-3}$ mbar OES gives lower absolute electron densities. The main limitation of this comparison at the present stage, is that the LP can not access the whole plasma chamber length, in particular it misses the low density plasma halos. Since OES averages over the whole plasma chamber length, including halos, OES density is expected to be lower than the LP one. For the electron 


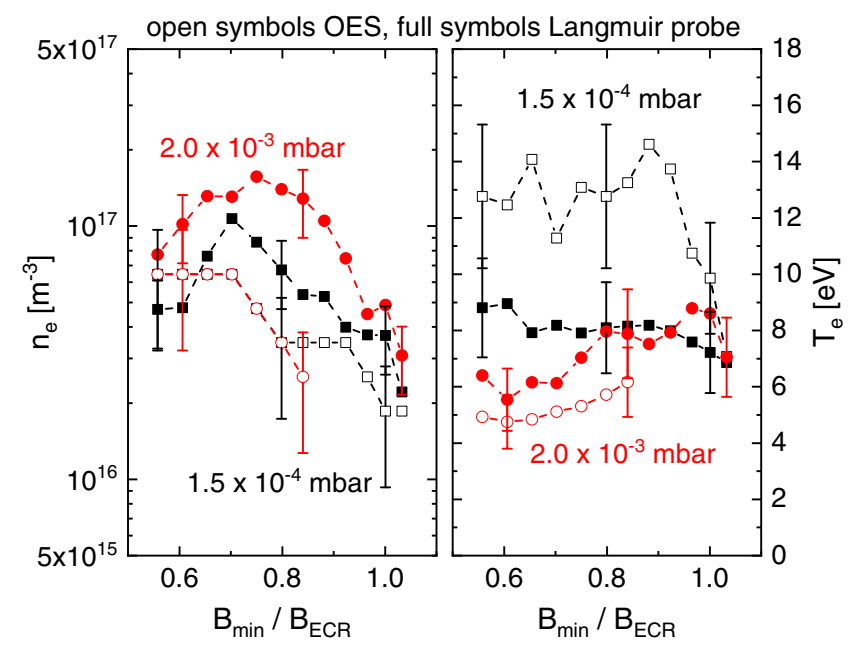

FIG. 8. Electron density and temperature calculated by means of LP and OES diagnostics versus $B_{\min } / B_{\mathrm{ECR}}$.

temperature obtained by LP and OES, the trends over $B_{\min } / B_{\mathrm{ECR}}$ agree well: at low pressure, no distinct variation is present, whereas at high pressure $T_{e}$ increases slightly. Concerning the absolute values, a good agreement is present for the $2.0 \times 10^{-3}$ mbar pressure case. At $1.5 \times 10^{-3}$ mbar, the electron temperature determined from OES is about $13 \mathrm{eV}$ but only around $8 \mathrm{eV}$ are measured via LP. This difference can be explained by the fact that these two diagnostics probe different energy regions of the EEDF: the LP is sensitive in the low energy range up of a few electron volts whereas OES is sensitive in the region above $10 \mathrm{eV}$ where the excitation energies of the atomic electronic states are located. If the EEDF is nonMaxwellian, the $T_{e}$ values obtained with the two diagnostics can deviate from each other. A selection of the x-ray

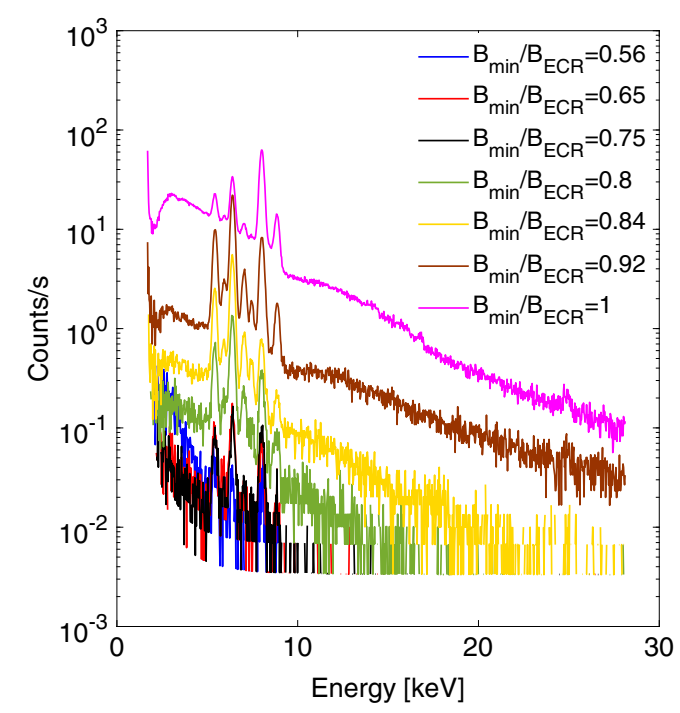

FIG. 9. X-ray spectra as a function of $B_{\min } / B_{\mathrm{ECR}}$ at $1.5 \times 10^{-4}$ mbar. spectra acquired by the SDD detector for different $B_{\min } / B_{\mathrm{ECR}}$ ratios are shown in Fig. 9. Spectra have been acquired only at $1.5 \times 10^{-4} \mathrm{mbar}$, since at $2 \times 10^{-3} \mathrm{mbar}$, no x-rays were detected by the SDD in the range of sensitivity of our setup. All the spectra have been corrected taking into account either the detector efficiency and the transmission through air and the kapton window. The presence of the characteristic emission lines of the materials composing plasma chamber implies the SDD cone of view intercepts the walls. Consequently, as anticipated in Sec. II D, absolute electron density cannot be evaluated, while the value of electron temperature is affected by electrons escaping from plasma core through the loss cone and hitting the walls. A quantitative estimation of the spectral slope, that we can define spectral temperature, is anyway important to give a rough trend of plasma energy vs the $B_{\min } / B_{\mathrm{ECR}}$ ratio variation. Due to the presence of characteristic emission lines, the spectral temperature evaluation has been in addition restricted to the energy domain 11-15 keV. X-ray emission rate and spectral temperature versus $B_{\min } / B_{\mathrm{ECR}}$ are shown in Fig. 10. A strong increase of the estimated spectral temperature and x-ray emission rate is visible when $B_{\min } / B_{\mathrm{ECR}}>0.8$, in the same conditions in which OES and LP diagnostics detected a decrease of electron density. These measurements permit to affirm that the energy content, as well as the number of the more energetic electrons, is increasing with $B_{\min } / B_{\mathrm{ECR}}$. In particular, results suggest that, when the threshold $B_{\min } / B_{\mathrm{ECR}}$ ratio is overcome, plasma energy content is transferred from cold electrons (temperature in the order of few eV) to warm and hot electrons (temperature $>1-10 \mathrm{keV})$. This phenomenon is probably due to the gradual change of the magnetic gradient at ECR, changing from strong gradient profile to a gentle gradient, as modeled by Canobbio [27] and numerically studied in Ref. [28].

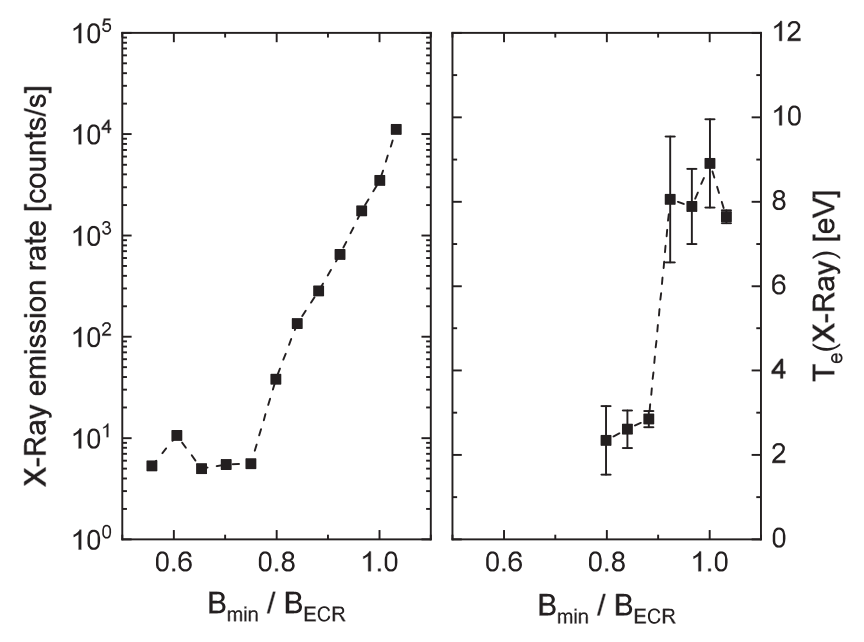

FIG. 10. X-ray emission rate and electron temperature for the $\mathrm{SDD}$ detector versus $B_{\min } / B_{\mathrm{ECR}}$. 


\section{CONCLUSION AND PERSPECTIVES}

The experimental results hereby presented represent one of the first characterizations in a multidiagnostics approach carried out in a compact plasma trap which closely emulates the behavior of an ECR ion source. Differently from last generation ECRIS, the machine used in the present paper consists of solely the axial confinement, provided by a simple mirror configuration. However, this has permitted to investigate the role played by solely the axial profile of the magnetic field. The scaling of electron density and temperature versus the $B_{\min } / B_{\mathrm{ECR}}$ ratio has been largely discussed. It has been experimentally found that, from the point of view of plasma density and "balance" of electrons in the cold or warm population, the optimal ratio lie in the range $0.7-0.75$. The density is lower at lower ratios, and it rapidly drops at $B_{\min } / B_{\mathrm{ECR}}>0.8$, when a simultaneous and dramatic increase of the plasma emission in the soft- $X$ domain has been observed. These results experimentally support the well-known scaling of ECRIS performances in terms of magnetic field tuning, demonstrating that the most relevant role is played by the axial field. Despite the present setup has been operated above the typical regimes characterizing highperformance ECRIS for multiply-charge ions, several experiments demonstrate the pressure is not the dominant parameter in this kind of discharges, unless we operate above $10^{-3}$ mbar. Therefore we deem that the outputs regarding the magnetic scaling remain valid even at lower pressure ranges.

The value of $B_{\min } / B_{\mathrm{ECR}} \sim 0.75-0.8$ can be considered as a threshold value, above which the EEDF is suddenly shifted toward the higher energy component. It is worthwhile to note that the diagnostics used in this work give back values of density and temperature averaged over the measurement time, which can be considered as a temporal resolution: LP averages over some ms, OES over seconds, and $\mathrm{x}$-ray detector over minutes. This means that the present diagnostics is unable to reveal oscillating behavior with significant variations of plasma parameters with resolution lower than the ms. Different independent measurements $[13,14,29,30]$ have shown that the same threshold value found in this paper plays a key role in establishing the conditions for Cyclotron-Maser instabilities. A future paper will discuss about time-resolved measurements able to show temporal evolution of plasma parameters in sub-ms temporal domain.

Beside the magnetic scaling, it is worth mentioning that the direct comparison between data coming from LP and OES are consistent with each other. It is not a trivial conclusion, since in small chambers and small-size plasmas the perturbation induced by the probe is relevant; on the other side, the estimation of density and temperature from OES is affected by the uncertainty in the correct definition of the cone-of-view, which in principle would require a precise estimation of the plasma size. Data are roughly consistent in terms of absolute values, but especially in terms of relative trends, since the conclusions about optimal magnetic field ratios come from both the diagnostics methods.

\section{ACKNOWLEDGMENTS}

The support of the 5th National Committee of INFN through the PANDORA experiment is gratefully acknowledged. The contributions of L. Allegra, S. Passerello, F. Chines, S. Marletta, A. Maugeri, A. Seminara, G. Pastore, and S. Vinciguerra have been essential for the present work.

[1] S. Briefi, S. Mattei, D. Rauner, J. Lettry, M. Q. Tran, and U. Fantz, Experimental benchmark of the NINJA code for application to the Linac4 $\mathrm{H}^{-}$ion source plasma, New J. Phys. 19, 105006 (2017).

[2] A. Girard and G. Melin, ECR ion sources today Physics, performance and technology, Nucl. Instrum. Methods Phys. Res., Sect. A 382, 252 (1996).

[3] C. Lyneis, D. Leitner, D. Todd, S. Virostek, T. Loew, A. Heinen, and O. Tarvainen, Measurements of bremsstrahlung production and $\mathrm{x}$-ray cryostat heating in VENUS, Rev. Sci. Instrum. 77, 03A342 (2006).

[4] S. Gammino, D. Mascali, L. Celona, F. Maimone, and G. Ciavola, Considerations on the role of the magnetic field gradient in ECR ion sources and build-up of hot electron component, Plasma Sources Sci. Technol. 18, 045016 (2009).

[5] G. Castro et al., Overdense plasma generation in a compact ion source, Plasma Sources Sci. Technol. 26, 055019 (2017).

[6] R. Rácz, S. Biri, Z. Perduk, and J. Pálinkás, First results on radial and azimuthal dependence of plasma parameters in a hexapole-trapped ECR discharge, AIP Conf. Proc. 2011, 020010 (2018).

[7] S. Biri, A. Valek, T. Suta, E. Takács, Cs. Szabó, L. T. Hudson, B. Radics, J. Imrek, B. Juhász, and J. Pálinkás, Imaging of ECR plasmas with a pinhole X-ray camera, Rev. Sci. Instrum. 75, 1420 (2004).

[8] D. Mascali, G. Torrisi, O. Leonardi, G. Sorbello, G. Castro, L. G. Celona, R. Miracoli, R. Agnello, and S. Gammino, The first measurement of plasma density in an ECRIS-like device by means of a frequency-sweep microwave interferometer, Rev. Sci. Instrum. 87, 095109 (2016).

[9] R. Rácz et al., Electron cyclotron resonance ion source plasma characterization by energy dispersive $\mathrm{x}$-ray imaging, Plasma Sources Sci. Technol. 26, 075011 (2017).

[10] R. Kronholm, T. Kalvas, H. Koivisto, and O. Tarvainen, Spectroscopic method to study low charge state ion and cold electron population in ECRIS plasma, Rev. Sci. Instrum. 89, 043506 (2018).

[11] S. Gammino and G. Ciavola, The role of microwave frequency on the high charge states buildup in the ECR ion sources, Plasma Sources Sci. Technol. 5, 19 (1996).

[12] D. Hitz, A. Girard, G. Melin, S. Gammino, G. Ciavola, and L. Celona, Results and interpretation of high frequency experiments at $28 \mathrm{GHz}$ in ECR ion sources, future prospects, Rev. Sci. Instrum. 73, 509 (2002). 
[13] I. Izotov, T. Kalvas, H. Koivisto, R. Kronholm, D. Mansfeld, V. Skalyga, and O. Tarvainen, Broadband microwave emission spectrum associated with kinetic instabilities in minimum-B ECR plasmas, Phys. Plasmas 24, 043515 (2017).

[14] R. Miracoli, G. Castro, L. Celona, S. Gammino, D. Mascali, M. Mazzaglia, E. Naselli, and G. Torrisi, Characterization of ECR plasma by means of radial and axial X-ray diagnostics, J. Instrum. 14, C01016 (2019).

[15] M. Mazzaglia et al., Study of the influence of magnetic field profile on plasma parameters in a simple mirror trap, J. Instrum. 13, C11014 (2018).

[16] S. Gammino et al., The Flexible Plasma Trap (FPT) for the production of overdense plasmas, J. Instrum. 12, P07027 (2017).

[17] F. F. Chen, Langmuir probes in RF plasma: Surprising validity of OML theory, Plasma Sources Sci. Technol. 18, 035012 (2009).

[18] I. D. Sudit and R. C. Woods, A study of the accuracy of various Langmuir probe theories, J. Appl. Phys. 76, 4488 (1994).

[19] J. E. Allen, R. L. F. Boyd, and P. Reynolds, The collection of positive ions by a probe immersed in a plasma, Proc. Phys. Soc. London Sect. B 70, 297 (1957).

[20] J. G. Laframboise, Ph.D. thesis, University of Toronto (Canada), 1966.

[21] F. F. Chen, J. D. Evans, and W. Zawalski, Calibration of Langmuir probes against microwaves and plasma oscillation probes, Plasma Sources Sci. Technol. 21, 055002 (2012).

[22] G. Torrisi, D. Mascali, G. Sorbello, G. Castro, L. Celona, and S. Gammino, Numerical and experimental characterization of RF waves propagation in ion sources magnetoplasmas, IEEE Trans. Antennas Propag. 67, 2142 (2019).

[23] S. Briefi, D. Rauner, and U. Fantz, Determination of the rotational population of $\mathrm{H}_{2}$ and $\mathrm{D}_{2}$ including high- $N$ states in low temperature plasmas via the Fulcher- $\alpha$ transition, J. Quant. Spectrosc. Radiat. Transfer 187, 135 (2017).

[24] D. Wünderlich and U. Fantz, Evaluation of state-resolved reaction probabilities and their application in population models for $\mathrm{He}, \mathrm{H}$, and $\mathrm{H}_{2}$, Atoms 4, 26 (2016).

[25] A. Gumberidze et al., Electronic temperatures, densities, and plasma x-ray emission of a $14.5 \mathrm{GHz}$ electroncyclotron resonance ion source, Rev. Sci. Instrum. 81, 033303 (2010).

[26] D. Mascali et al., X-ray spectroscopy of warm and hot electron components in the CAPRICE source plasma at EIS testbench at GSI, Rev. Sci. Instrum. 85, 02A956 (2014).

[27] E. Canobbio, Gyroresonant particle acceleration in a nonuniform magnetostatic field, Nucl. Fusion 9, 27 (1969).

[28] S. Gammino, D. Mascali, L. Celona, F. Maimone, and G. Ciavola, Considerations on the role of the magnetic field gradient in ECR ion sources and build-up of hot electron component, Plasma Sources Sci. Technol. 18, 045016 (2009).

[29] D. Mascali et al., An investigation on the formation of suprathermal electrons in a $B$-min ECR machine and a novel method for their damping, Plasma Sources Sci. Technol. 22, 065006 (2013).

[30] D. Neben, X-ray investigation on the Superconducting Source for Ions (SuSI), J. Instrum. 14, C02008 (2019). 